\title{
A Novel Field Test to Determine Critical Speed
}

\section{Galbraith A, Hopker JG*, Jobson SA and Passfield L}

Centre for Sports Studies, University of Kent, Kent, England

\begin{abstract}
The aim of this study was to assess the reliability of a novel field test of critical running speed (CS). Ten trained male distance runners completed a familiarisation trial plus three separate experimental trials on a standard $400 \mathrm{~m}$ athletics track. Each trial consisted of three distances (1200, 2400 and 3600 metres) that were selected to produce finishing times in the region of 3,7 and 12 minutes respectively. Participants were instructed to cover the set distance in the fastest time possible. Participants rested for 30 minutes between efforts. Data were modelled using the linear distance-time model, described by the equation: $d=(C S \times t)+A R C$, where: $d=$ distance run $(m), t=$ running time $(\mathrm{s})$, and ARC = anaerobic running capacity $(\mathrm{m})$. Results demonstrated a coefficient of variation $(\mathrm{CV})$ of $2.0 \%(95 \%$ confidence limit $(95 \% \mathrm{CL}): 1.4-3.8 \%)$ for trials $2-1$ and $1.3 \%(95 \% \mathrm{CL}: 0.9-2.4 \%)$ for trials $3-2$. There was no significant difference in CS $\left(\mathrm{m} \cdot \mathrm{s}^{-1}\right)$ across trials $(P<0.05)$. The limits of agreement were $\pm 0.27 \mathrm{~m} \cdot \mathrm{s}^{-1}$ of the measure for trials 2-1 and $\pm 0.18 \mathrm{~m} \cdot \mathrm{s}^{-1}$ for trials 3-2. ARC proved to be less reliable with a group CV of $18.4 \%$ (95\% CL: $13.5-$ $39.9 \%$ ) for trials $2-1$ and $9.8 \%$ (95\% CL: $7.0-19.6 \%)$ for trials 3-2. Although the assessment of ARC is less reliable, $\mathrm{CV}$ data are similar to those reported previously during laboratory-based testing.
\end{abstract}

Keywords: Critical velocity; Endurance; Running, Economy; Anaerobic work capacity

\section{Introduction}

It has been suggested that the Critical Power (CP) demarcates the heavy and severe exercise domains [1] and as such corresponds to an exercise intensity which lies between that associated with the lactate threshold and that eliciting $\dot{\mathrm{V}}_{2 \max }$ [2]. Consequently, $\mathrm{CP}$ has been associated with overall athletic performance in long-duration events $[3,4]$. The concept of CP has been applied to treadmill running [5], that is, where the relation between treadmill running velocity and time to exhaustion conforms to a hyperbolic function similar to that seen in cycling. This relationship has traditionally been termed Critical Velocity, however as the present study utilised a field test where subjects were required to run a set number of laps of an athletics track, Critical Speed (CS) is a more appropriate term. Therefore, to allow standardisation of terminology CS will be used for the remainder of the paper, regardless of whether the reference is to treadmill or field testing.

Many early 'critical power' studies calculated CP by plotting the total work done against the time taken to complete that work. For running exercise this model has been transformed into a distancetime model, where the total distance covered was plotted against the time taken to cover that distance [6]. This transformation of the CP model can be described by a linear relationship, where the slope of the regression line calculates $C S$ and the $y$-intercept is termed anaerobic running capacity (ARC).

The traditional method of testing CS in a laboratory involves athletes completing a set number of time-to-exhaustion (TTE) trials at a constant speed on the treadmill. Constant speed trials have been shown to have poor reliability with coefficients of variation ranging from $15.1 \%$ to $25 \%[7,8]$. This is supported by similar research in both cycling and swimming, which also demonstrated the poor reliability of constant power/speed trials $[9,10]$. However, research into the reliability of the CS and ARC parameters is limited. Hinckson and Hopkins (2005) looked at the reliability of CS and ARC measured on a treadmill. They demonstrated good reliability of CS data (coefficient of variation 1.8\%), but poor reliability of ARC data (coefficient of variation 14\%). These researchers used constant speed trials where participants were required to run to exhaustion at three pre-set constant speeds that resulted in exhaustion times of approximately $1-2,3-4$ and $7-10$ minutes.
Constant distance trials, where the athlete is required to cover a set distance in the fastest possible time, have been shown to have a far better reliability, with coefficients of variation ranging from $3.3 \%$ to $3.7 \%[7,11]$. Due to the limitations of the manual speed control measures on standard motorised treadmills, such trials are arguably best performed in a field-based setting. However, there appears to be no research on the reliability of CS and ARC using constant distance trials. Therefore, the purpose of the current study was to assess the reliability of CS and ARC determined in the field on an athletics track.

\section{Materials and Methods}

\section{Subjects}

Following institutional ethics approval, ten trained male middle distance runners (age: $22 \pm 4 \mathrm{yrs} ; \dot{\mathrm{VO}}_{2 \max } 69.1 \pm 4.2 \mathrm{~mL} \cdot \mathrm{kg}^{-1} \cdot \mathrm{min}^{-1}$ ) were recruited for the study. All athletes were competitive club or national standard runners who had been competing for a minimum of 2 years. Subjects refrained from heavy exercise in the 24 hours prior to all tests and from food in the 3 hours prior to all tests. Tests for individual subjects were completed at the same time of day to eliminate a possible effect of circadian rhythms [12].

\section{Experimental design}

Each subject completed five experimental visits. At visit 1 , subjects completed an incremental exercise test to determine $\mathrm{VO}_{2 \max }$, whilst during visit 2 subjects completed a familiarisation of the field test protocol. During visits 3, 4 and 5 subjects completed repeated tests of the field test protocol.

*Corresponding author: James G. Hopker, Centre for Sports Studies, University of Kent, Kent, ME4 4AG, England, Tel: +44 16348888 14; Fax: +44 16348888 90; E-mail: jgh@kent.ac.uk

Received September 04, 2011; Accepted October 28, 2011; Published October 31, 2011

Citation: Galbraith A, Hopker JG, Jobson SA, Passfield L (2011) A Novel Field Test to Determine Critical Speed. J Sport Medic Doping Studie 1:101. doi:10.4172/21610673.1000101

Copyright: (c) 2011 Galbraith A, et al. This is an open-access article distributed under the terms of the Creative Commons Attribution License, which permits unrestricted use, distribution, and reproduction in any medium, provided the original author and source are credited. 


\section{Determination of subject characteristics}

Subjects completed a 5-min self-paced warm-up [13], on an $\mathrm{H} / \mathrm{P} /$ Cosmos Saturn 4.0 treadmill (H/P/Cosmos Sports and Medical, Nussdorf-Traunstein, Germany) set to a $1 \%$ gradient as recommended by Jones and Doust (1996). Following a 5-min self-selected stretching routine, subjects completed a two-phase protocol [14] to determine running economy $\left(\mathrm{mL} \cdot \mathrm{kg}^{-1} \cdot \mathrm{km}^{-1}\right), \mathrm{VO}_{2 \max }$, and velocity at $\mathrm{VO}_{2 \max }(\mathrm{v}$ $\mathrm{V}_{2 \max }$ ). At the end of each 3 -min stage during phase 1 , treadmill speed was increased by $1.0 \mathrm{~km} \cdot \mathrm{h}^{-1}$. Phase 1 of the protocol was terminated when the subject reached a lactate concentration $>4.0$ mmol. $\mathrm{L}^{-1}$. Following a 15 -minute recovery, the second phase of the test was initiated at a speed $2.0 \mathrm{~km} \cdot \mathrm{h}^{-1}$ below the speed at which the subject completed phase 1 . Whilst treadmill speed remained constant throughout phase 2 of the protocol, treadmill gradient was increased by $1 \%$ every minute until volitional exhaustion. Pulmonary gas exchange was measured breath-by-breath (MetaLyser 3B, Cortex Biophysik, Leipzig, Germany). $\dot{\mathrm{VO}}_{2 \max }$ was recorded as the highest mean oxygen consumption over a 60 -s period.

\section{Determination of critical speed}

Critical Speed was calculated from three constant distance runs $(3600,2400,1200 \mathrm{~m})$ carried out on a competition standard $400 \mathrm{~m}$ outdoor running track. These distances were estimated to yield finishing times between 2 and $12 \mathrm{~min}$ [5]. Testing was only carried out when wind speed was less than $2.0 \mathrm{~m} \cdot \mathrm{s}^{-1}$ [15]. Subjects completed a standardised warm-up (5-min self-paced jogging, followed by a 5-min stretching routine). Subjects were then instructed to cover the set distance in the fastest time possible. Finishing times for the three distances were recorded to the nearest second. All three runs were conducted in the order of longest to shortest, on the same day, with a 30-min rest between them. Linear regression was used to calculate CS and ARC from the results of these trials using the $\mathrm{d}=(\mathrm{CS} \times \mathrm{t})+\mathrm{ARC}$ model, where: $\mathrm{d}=$ distance run $(\mathrm{m}), \mathrm{CS}=$ critical running speed $\left(\mathrm{m} \cdot \mathrm{s}^{-1}\right), \mathrm{t}=$ running time $(\mathrm{s})$, and $\mathrm{ARC}=$ anaerobic running capacity $(\mathrm{m})$.

\section{Data analysis}

Data were assessed for normality of distribution. To assess the stability reliability of CS and ARC, the within-subject variation, expressed as a coefficient of variation (CV), was derived from log-transformed data [16]. The 95\% confidence intervals were calculated for each CV. Confidence intervals (95\% CI) of the CV and 95\% limits of agreement were calculated per participant to assess the variability of the repeated tests [16]. Comparisons of CS and ARC across days were assessed using repeated measures ANOVA. Statistical significance was set at $95 \%$ confidence $(\mathrm{P}<0.05)$. Results are reported as mean \pm SD unless otherwise stated.

\section{Results}

A mean group typical error, expressed as a coefficient of variation, of $2.0 \%$ (95\% confidence limit (95\% CL): $1.4-3.8 \%$ ) for trials $2-1$ and $1.3 \%$ (95\% CL: $0.9-2.4 \%$ ) for trials $3-2$ was found. There was no significant difference in CS across trials $(\mathrm{P}<0.05)$. Repeated measures ANOVA also confirmed the absence of an order effect in the data. The limits of agreement were $\pm 0.27 \mathrm{~m} \cdot \mathrm{s}^{-1}$ of the measure for trials $2-1$ and $\pm 0.18 \mathrm{~m} \cdot \mathrm{s}^{-1}$ for trials $3-2$, (Figure 1 ). ARC proved to be less reliable with a group CV of $18.4 \%$ (95\% CL: $13.5-39.9 \%$ ) for trials $2-1$ and $9.8 \%$ (95\% CL: 7.0-19.6\%) for trials 3-2, (Figure 2), although this variability did not result in significant differences between trials $(\mathrm{P}>0.05)$.

Based on a mean CS of $4.72 \mathrm{~m} . \mathrm{s}^{-1}$ and the mean CV for CS of $1.7 \%$, an athlete would have to improve their CS by $0.08 \mathrm{~m} . \mathrm{s}^{-1}$ in order to detect a meaningful change in performance. Theoretically this could be achieved by an improvement of just over 1 second per lap during the constant distance trials.

\section{Discussion}

The results of the current study demonstrate that critical speed can be reliably tested using a novel same day field test. The mean CV of $1.7 \%$ is similar to that previously reported in the literature [17] for laboratory based testing of CS. A 5\% coefficient of variation has been cited as an acceptable upper limit in sports science reliability studies [18]. Given that the CV values observed were below this boundary we might consider CS from the novel field test to be reliable. In agreement with previous literature [17] ARC proved to be less reliable, with a CV of $14.1 \%$. Therefore, the CS and ARC reliability results of the current study are similar to those reported previously during laboratorybased testing. However, such a level of variation in ARC is unlikely to be acceptable when evaluating the relatively small training-induced changes seen in well-trained athletes [16]. Such a conclusion is supported by limits of agreement analyses which suggest that, with an approximate $95 \%$ probability, the differences between the test and retest of ARC in an individual from the well-trained running population will at best, lie between $\pm 48 \mathrm{~m}$.

Assuming that the bias is negligible, ratio limits of agreement suggest that, between any two tests, CS may typically differ by $4.7 \%$ and ARC by $39.0 \%$, in a positive or negative direction. The coefficient of variation for both CS and ARC decreased from trials 2-1 to trials $3-2$, although there were no significant differences in CS or ARC across trials $(\mathrm{P}<0.05)$. These results suggest the need for several familiarisation trials before using the novel constant distance field trial to monitor performance.

Most of the previous literature investigating CS has required a subject to run at a set speed until exhaustion. These have traditionally been shown to have poor reliability with coefficients of variation ranging from 15.1 to $25 \%[7,8]$ Similar findings have been reported in both cycling [9] and swimming [10]. Hinckson and Hopkins [17] used a variety of approaches to produce estimates of test-retest error of measurement calculated from times to exhaustion. All reliability estimates were $<3 \%$, and some were $\sim 1 \%$, resulting in the authors stating that their findings should lay to rest any concerns that time to exhaustion is inherently an unreliable measure of endurance performance.

A major criticism can be levelled at the use of constant speed trials in testing CS, typically performed on a treadmill. These trials are not ecologically valid, and do not mimic any training or race situation for a competitive athlete. In training and racing athletes are required to cover a set distance in the fastest time possible, and are rarely (if ever), required to run at a constant speed until exhaustion. In the current study we decided to take the more ecologically valid approach of using constant distance trials. Even so, one disadvantage of this approach is the potential influence of pacing. The potential impact of poor pacing strategy was decreased in the current study by the selection of trained distance runners as participants. However, alterations in pacing might indicate why the CV decreased over the time course of the repeated experimental trials.

A novel aspect of the constant distance field trial used in the present study was that each of the individual runs used to model CS and ARC were completed with a 30-min recovery period between them. This allowed the whole testing session to be completed within 

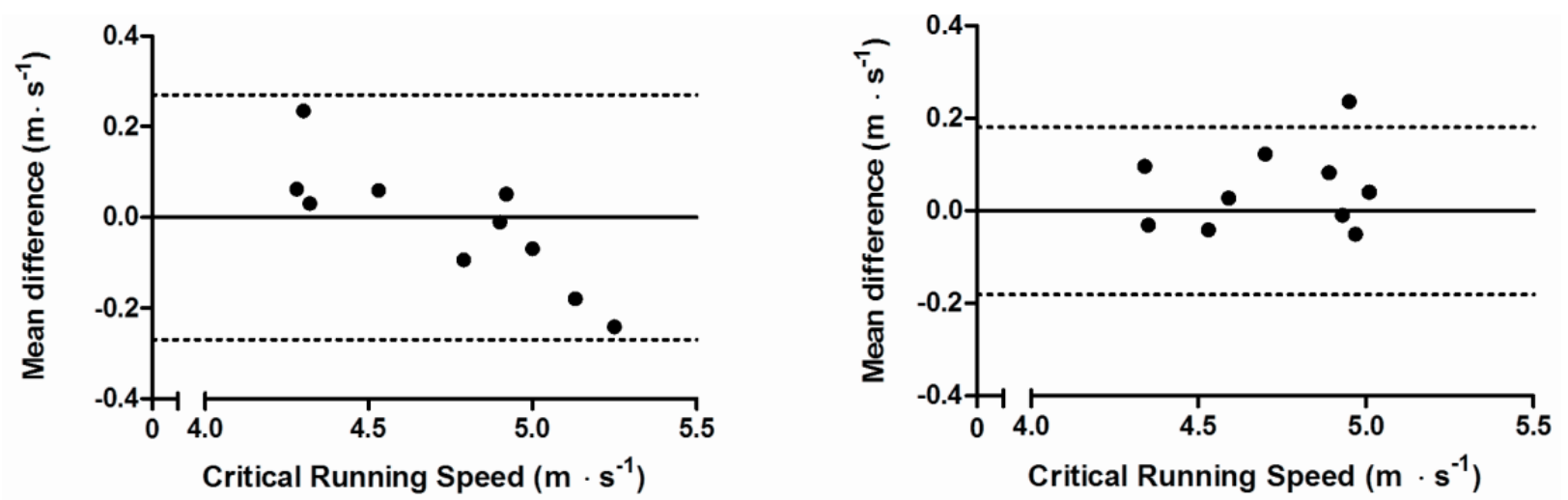

Figure 1: Bland-Altman plots of the Critical Running Speed test-re-test differences between trials 1 and 2 [left] and trials 2 and 3 [right]. The solid horizontal lines represent mean bias, whilst the dashed lines represent the $95 \%$ limits of agreement.
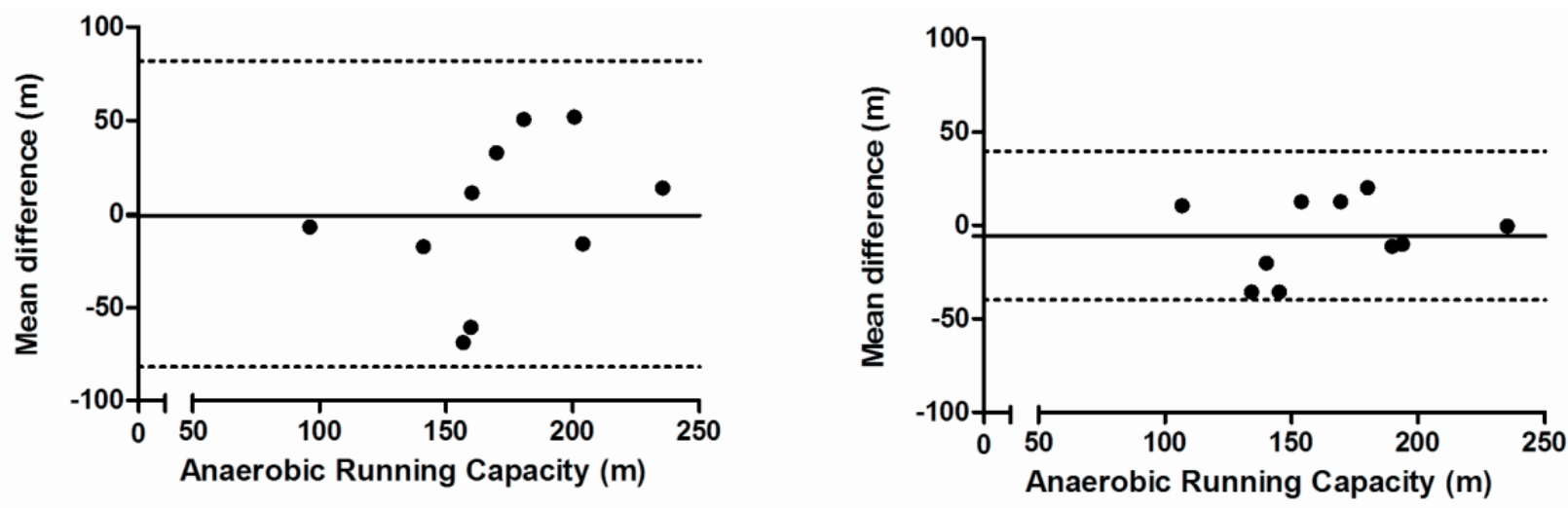

Figure 2: Bland-Altman plots of the Anaerobic Running Capacity test-re-test differences between trials 1 and 2 [left] and trials 2 and 3 [right]. The solid horizontal lines represent mean bias, whilst the dashed lines represent the $95 \%$ limits of agreement.

a 2-hour time frame. Traditionally when CS and ARC are tested in a laboratory on a treadmill, recovery periods in excess of 24 hours are commonly used $[13,19]$ making this a protracted approach. The results of the current study demonstrate that the constant distance field trial is a reliable method of assessing CS and ARC that may present a more attractive option to sports scientists, athletes and coaches wishing to monitor physical fitness, endurance performance, and design optimal pacing strategies.

Using the novel field test a coach could gain information on an athlete's aerobic and anaerobic capabilities, from the CS and ARC parameters respectably. As the testing procedure takes a relatively short length of time, these parameters could be monitored at regular intervals through a season to assess the impact of training on CS and ARC. Previous research in cycling has indicated that Critical Power increases following a period of interval endurance training [20,21], and Anaerobic Work Capacity increases following a period of power or sprint training [22].

Using the following equation the distance-time relationship can be used to calculate the quickest time in which an athlete could complete a set distance:

$$
\mathrm{t}=(\mathrm{D}-\mathrm{ARC}) / \mathrm{CS}[23]
$$

Where $\mathrm{t}=$ predicted time taken to complete a set distance and $\mathrm{D}=$ the chosen set distance.
This prediction of performance could provide a runner with a realistic target to aim for in competitive races. Predicted performance from the distance-time relationship has shown good correlation with actual performance over distances ranging from 10,000m [24] to the Marathon (Florence and Weir, 1997).

An athlete and their coach could also use information obtained from the distance-time relationship to formulate pacing and tactical strategies aimed at maximizing competitive performance [25]. For example, in a competitive race situation the best tactical pacing strategy for an athlete with a relatively low CS but a high ARC, might be to slow the pace and use their high ARC to full effect in a sprint finish [25]

Finally, it has been suggested that the distance-time relationship can be used to rank runners in terms of ability. Successful athletes not only need a high $\mathrm{VO}_{2 \max }$, but also the ability to sustain a high percentage of this value for the duration of their event - i.e. they need good aerobic endurance. Gamelin et al. (2006) suggest that CS takes into account both $\mathrm{VO}_{2 \max }$ and aerobic endurance. Therefore, they suggest that CS should be used to rank middle and long distance runners with regard to their ability in long-distance running events.

\section{Conclusion}

The results of the current study demonstrate that a novel constant distance field trial reliably assesses CS and produces reliability data comparable to that previously reported using constant speed trials. Al- 
though the assessment of ARC is less reliable, coefficients of variation are also similar to those reported previously during laboratory-based testing. Therefore, the novel constant distance field trial could be used as a suitable, more ecologically valid alternative to treadmill based constant speed trials when assessing CS and ARC.

\section{References}

1. Bull AJ, Housh TJ, Johnson GO, Rana SR (2008) Physiological responses a five estimates of critical velocity. Eur J Appl Physiol 102: 711-720.

2. Billat V, Binsse V, Petit B, Koralsztein JP (1998) High level runners are able to maintain $\mathrm{a} \mathrm{VO}_{2}$ steady-state below $\mathrm{VO}_{2 \text { max }}$ in an all-out run over their critical velocity. Arch Physiol Biochem 106: 38-45.

3. Housh TJ, Devries HA, Housh DJ, Tichy MW, Smyth KD, et al. (1991) The relationship between critical power and the onset of blood lactate accumulation. J Sports Med Phys Fitness 31: 31-36.

4. Jenkins DG, Quigley BM (1990) Blood lactate in trained cyclists during cycle ergometry at critical power. Eur J Appl Physiol Occup Physiol 61: 278-283.

5. Hughson RL, Orok CJ, Staudt LE (1984) A high velocity treadmill running test to assess endurance running potential. Int J Sports Med 5: 23-25.

6. McDowell S, Kenney K, Hughes R, Housh T, Johnson G (1988) The relationship between ventilatory threshold and critical velocity. AAHPERD Publications Kansas City.

7. Laursen PB, Francis GT, Abbiss CR, Newton MJ, Nosaka K (2007) Reliability of time-to-exhaustion versus time-trial running tests in runners. Med Sci Sports Exerc 39: 1374-1379

8. Billat V, Renoux JC, Pinoteau J, Petit B, Koralsztein JP (1994) Reproducibility of running time to exhaustion at $\mathrm{VO}_{2 \max }$ in subelite runners. Med Sci Sports Exerc 26: 254-257.

9. Jeukendrup A, Saris WH, Brouns F, Kester AD (1996) A new validated endurance performance test. Med Sci Sports Exerc 28: 266-270.

10. Alberty M, Sidney M, Huot-Marchand F, Dekerle J, Bosquet L, et al. (2006) Reproducibility of performance in three types of training test in swimming. Int $J$ Sports Med 27: 623-628.

11. Nicholson RM, Sleivert GG (2001) Indices of lactate threshold and their relationship with 10-km running velocity. Med Sci Sports Exerc 33: 339-342.
12. Drust B, Waterhouse J, Atkinson G, Edwards B, Reilly T (2005) Circadian rhythms in sports performance--an update. Chronobiol Int 22: 21-44.

13. Smith CG, Jones AM (2001) The relationship between critical velocity, maximal lactate steady-state velocity and lactate turnpoint velocity in runners. Eur J App Physiol 85: 19-26.

14. Jones AM (2008) Middle and long-distance running. In Winter EM, Jones AM Davison RCR, Bromley PD, Mercer TH (Eds.) Sport and exercise physiology testing guidelines. volume one: Sport testing (pp. 147-154). Routledge, Oxford.

15. Jones AM, Doust JH (1996) A 1\% treadmill grade most accurately reflects the energetic cost of outdoor running. J Sports Sci 14: 321-327.

16. Hopkins WG (2000) Measures of reliability in sports medicine and science. Sports Med 30: 1-15.

17. Hinckson EA, Hopkins WG (2005) Reliability of time to exhaustion analyzed with critical-power and log-log modeling. Med Sci Sports Exerc 37: 696-701.

18. Hopkins WG (2000b) A new view of statistics [Internet]. Internet Society for Sport Science [cited 2011 August 16].

19. Hill DW, Ferguson CS (1999) A physiological description of critical velocity. Eur J Appl Physiol Occup Physiol 79: 290-293.

20. Jenkins DG, Quigley BM (1992) Endurance training enhances critical power Med Sci Sports Exerc 24: 1283-1289.

21. Vanhatalo A, Doust JH, Burnley M (2008) A 3-min all-out cycling test is sensitive to a change in critical power. Med Sci Sports Exerc 40: 1693-1699.

22. Jenkins DG, Quigley BM (1993) The influence of high intensity exercise training on the Wlim-Tlim relationship. Med Sci Sports Exerc 25: 275-282.

23. Gaesser GA, Carnevale TJ, Garfinkel A, Walter DO, Womack CJ (1995) Estimation of critical power with nonlinear and linear models. Med Sci Sports Exerc 27: 1430-1438.

24. Kranenburg KJ, Smith DJ (1996) Comparison of critical speed determined from track running and treadmill tests in elite runners. Med Sci Sports Exerc 28 614-618.

25. Jones AM, Vanhatalo A, Burnley M, Morton RH, Poole DC (2010) Critica Power: Implications for determination of $\mathrm{VO}_{2}$ and exercise tolerance. Med Sci Sports Exerc 42: 1876-1890. 